\title{
TESTE DE UM MODELO CAUSAL ENTRE VALORES HUMANOS E CONDUTAS DESVIANTES EM JOVENS
}

\author{
SCALE TEST OF A CAUSAL MODEL BETWEEN HUMAN VALUES AND DEVIANT BEHAVIOUR \\ OF YOUNG PEOPLE
}

Recibido: 15 de Abril del 2011 | Aceptado: 28 de Abril del 2011

\section{NILTON S. FORMIGA,}

(UNIVERSIDADE FEDERAL DA PARAÍbA, Faculdade Mauricio de Nassau, Brasil).

\begin{abstract}
RESUMEN
Neste trabalho pretende verificar um modelo teórico no qual se hipotetiza associação dos valores humanos e as condutas antissociais e delitivas. Neste caso, os valores de orientação social - coletivista - explicaria negativamente as condutas deviantes, por outro lado, a orientação valorativa pessoal - individualista - se associará positivamente as condutas desviantes. 504 jovens, do sexo masculino e do sexo feminino, de 15 e 22 anos, participaram do estudo. Responderam a Escala de condutas Antissociais e delitivas, 0 Questionário dos valores humanos básicos e questões sócio-demográficas. No programa AMOS GRAFICS 18.0, gerou-se o modelo hipotetizado, observando uma associação negativa entre os valores sociais e as condutas desviantes e positiva entre valores pessoais e as mesmas condutas.
\end{abstract}

PALABRAS CLAVE: Modelo teórico, Condutas desviantes, Valores humanos, Jovens.

\section{ABSTRACT}

This work aims to verify a theoretical model in which hypothesized the association of human values and antisocial and criminal behavior. In this case, the values of social orientation - collectivist - explain negatively the deviant behavior; on the other hand, the personal value orientation - individualist - is positively associated with deviant behavior. 504 young, male and female, between 15 and 22 years old participated in this study. They answered the antisocial and criminal behavior Scale, the Questionnaire about basic human values and social demographics. The program AMOS GRAFICS 18.0, created a hypothesized model, observing a negative association between social values and deviant behaviors and positive relationship between personal values and the same conduct.

KEY WORDS: Theoretical model, Deviant behavior, Human values, Young people.

\footnotetext{
1. Nilton S. Formiga, Doutor em Psicologia Social pela Universidade Federal da Paraíba; atualmente é professor no curso de Psicologia na Faculdade Mauricio de Nassau. Endereço para correspondência: Rua Juiz Ovídio Gouveia, 349. Pedro Gondim. CEP.: 58031-030. João Pessoa - PB. E-mail: nsformiga@yahoo.com.
} 
INTRODUCCIÓN

A explicação do comportamento desviante entre os jovens tem contemplado variáveis estruturais e funcionais com o objetivo de responder as causas e os efeitos desse comportamento na dinâmica psicológica e social do jovem e seu entorno ambiental (Agüero, 1998; Muñoz-Rivas \& Graña, 2002; Stoff, Breiling \& Maser, 1997; Sukhodolsky, Colub \& Cromwell, 2001). Acredita-se que 0 problema da conduta desviante juvenil, atualmente, não tem um padrão invariável atribuindo-lhe exclusividade, de um grupo social em função da variação sócioeconômica e demográfica, bem como, conseqüente de um distúrbio de personalidade (Cava, Buelgas, Herrero \& Lilá, 2000; Formiga \& Gouveia, 2005; Formiga, 2010; Torrente \& Rodríguez, 2000).

Esse fenômeno na época atual é um reflexo de uma mudança cultural que encontrada em muitos países ocidentais fomentadores de um espírito individualista, subordinando interesses e prioridades pessoais ao invés daqueles do grupo e da harmonia social. A meta desses jovens é, somente, buscar prestígio e satisfazer seu próprio ego e imagem pessoal, pois, quando não conseguem alcançar esse objetivo ou quando na falta de recursos econômicos ou apoio social, geralmente, procuram obter 0 que desejam através de uma conduta delinqüente (Formiga \& Diniz, 2011; Lipovetsky, 1986).

Frente a tais reflexões, o problema da conduta desviante entre os jovens, explorado por pesquisadores em diversos espaços da ciência humana e social, tem como objetivo apresentar soluções a fim de controlar ou amenizar esse fenômeno. Dessas soluções, uma que tem sido considerada importante, refere-se aos fatores psicossociais; neste contexto, pretende-se enfatizar os aspectos normativos de tais condutas, isto é, o compromisso com os padrões convencionais estabelecidos na sociedade, destacando o papel dos valores humanos; observa-se que uma adesão a valores mais pessoais (por exemplo, emoção, prazer) promove esse tipo de conduta, enquanto uma adesão a valores sociais (por exemplo, religiosidade, tradição) é capaz de inibir tais condutas (Coelho Júnior, 2001; Formiga \& Gouveia, 2005; Petraitis, Flay \& Miller, 1995; Romero, Sobral, Luengo \& Marzoa, 200; Tamayo, Nicaretta, Ribeiro \& Barbosa, 1995).

Com base na suposição teórica de que os valores humanos assumem a natureza benevolente ou positiva dos seres humanos, de que servem como padrões gerais de orientação para os comportamentos dos indivíduos, de que são representações cognitivas das necessidades humanas, necessidades estas não só individuais, mas também de ordem institucionais e societais e de que se compreendem como metas superiores estando além de metas imediatas e biologicamente urgentes (Gouveia, Milfont, Fischer \& Santos, 2008), este construto é considerado como "conceitos ou categorias; sobre estados desejáveis de existência; transcendem situações específicas; assumem diferentes graus de importância; guiam a seleção ou avaliação de comportamentos e eventos e representam cognitivamente as necessidades humanas" (p.55).

Nesta teoria dos valores, este construto, é considerado como critério de orientação que guiam as ações do homem e expressam as suas necessidades básicas; tem-se como sua primeira função guia o tipo de orientação social, central e pessoal. Os indivíduos que são guiados por valores sociais centram-se na sociedade e foco interpessoal, inseridos na subfunção Normativa (que se refere com orientações dentro do domínio motivador materialista) e Interacional (diz respeito às orientações sociais dentro do domínio motivador humanitário); por outro lado, aquelas pessoas que são guiadas por valores pessoais, são egocêntricos ou tem um foco 
intrapessoal, contemplando a subfunção de Experimentação (compatível com orientações pessoais dentro do domínio motivador humanitário) e Realização é compatível com orientações pessoais dentro do domínio motivador materialista).

A segunda função dos valores expressa às necessidades básicas do homem aponta que indivíduos guiados por tais valores tendem a pensar em condições de sobrevivência mais físicas, ressaltando a sua própria existência e as condições de mantê-las, identificando o tipo de motivação materialista (pragmático) ou humanitário (idealista) do sujeito que se guia por essa adesão. De acordo com Gouveia, Milfont, Fischer e Santos (2008), as subfunções dos valores, em graus diferentes, enfatizam 0 ajuste do indivíduo à sociedade e às suas instituições, mas também acentuam a sobrevivência do indivíduo. Entretanto, essas funções valorativas e suas respectivas subfunções são estruturas latentes, que precisam ser representadas por variáveis observáveis, tais como indicadores, itens ou valores específicos.

Ao considerar a hipótese, especificamente, a de que os valores humanos explicam as condutas desviantes, estas, são compostas pela conduta antissocial e deltiva, Formiga (2002) e Formiga e Gouveia (2005; Formiga; Gouveia, 2006) desenvolveram um estudo correlacional com 710 jovens do sexo feminino e do sexo masculino na cidade de João Pessoa - PB, com idade de 15 a 20 anos. Nesses três estudos, os autores observaram que os jovens que dão mais importância aos valores pessoais apresentaram mais indícios tanto de condutas anti-sociais quanto delitivas, enquanto que aqueles que deram importância os valores sociais foram menos prováveis em relatar tais condutas. Resultados semelhantes, porém, considerando a variável o uso potencial de drogas, foram encontrados por Coelho Júnior (2001).
Apesar desses autores brasileiros (por exemplo, Coelho Júnior, 2001; Formiga, 2002; Formiga \& Gouveia, 2005; Formiga \& Gouveia, 2006) encontrarem resultados consistentes tanto com base na perspectiva teórica assumida quanto na operacionalidade de mensurar das condutas desviantes e dos valores humanos, ao considerar apenas esse tipo de análise correlacional aponta-se em direção de um inconveniente: esta análise pauta-se estritamente nos dados obtidos não considerando um modelo teórico fixo que oriente a extração das dimensões latentes e muito menos têm o poder de apresentar qualquer indicação sobre a bondade de ajuste do modelo. Estas técnicas - a da análise da modelagem estrutural - têm a clara vantagem de levar em conta a teoria para definir a associação entre as variáveis hipotetizadas, bem como, apresenta indicadores de bondade de ajuste que permitem decidir objetivamente sobre a validade de construto da medida analisada.

Dois resultados principais podem ser esperados ao trabalhar com a análise estrutural: 1) estimativa da magnitude dos efeitos estabelecida entre variáveis, as quais estão condicionadas ao fato de 0 modelo especificado (isto é, o diagrama) estar correto, e 2) testar se o modelo é consistente com os dados observados, a partir dos indicadores estatísticos, podendo dizer que resultado, modelo e dados são plausíveis, embora não se possa afirmar que este é correto (Farias \& Santos, 2000). Atende-se assim, não a certeza total do modelo, mas, a sua probabilidade sistemática na relação entre as variáveis.

Um dos principais objetivos das técnicas multivariadas - neste caso, considera-se a modelagem de equação estrutural - é expandir a habilidade exploratória do pesquisador e a eficiência estatística e teórica no momento em que se quer provar a hipótese levantada no estudo. Apesar das técnicas estatísticas tradicionais compartilharem de limitações, nas quais, é 
possível examinar somente uma relação entre as variáveis, é de suma importância para o pesquisador o fato de ter relações simultâneas; afinal, em alguns modelos existem variáveis que são independentes em algumas relações e, dependentes em outras.

A fim de suprir esta necessidade, a Modelagem de Equação Estrutural examina uma série de relações de dependência simultâneas, esse método é particularmente útil quando uma variável dependente se torna independente em relações subseqüentes de dependência (Silva, 2006; Hair, Anderson, Tatham \& Black, 2005). Com isso, o objetivo do presente estudo trata-se da avaliar de forma mais robusta, a partir de uma Análise Fatorial Confirmatória $(A F C)$ e a análise do Modelo de Equação Estrutural (SEM) efetuado a partir do AMOS GRAFICS, versão 18.0, a associação entre os valores humanos e as condutas desviantes (antisocial e delitiva) em uma amostra de jovens paraibanos. Para isso toma-se como referência o estudo de Formiga e Gouveia (2005; Formiga \& Gouveia, 2006), a partir de uma estatística mais sofisticada, têm-se como base as mesmas hipóteses elaboradas por esses autores pretendendo-se, com isso, corroborar os seus achados, a saber:

H1 - espera-se que a orientação valorativa social se associe, inversamente, com as condutas desviantes (composta pela conduta antissocial e delitiva);

H2 - por outro lado, espera-se que a orientação valorativa pessoal associar-se-á, diretamente, com as condutas desviantes.

\section{MÉTODO}

\section{Amostra}

504 jovens, do sexo masculino e feminino, com idades de 14 a 20 anos, distribuído igualmente nos níveis escolares fundamental e nível médio, da rede privada e pública de educação da cidade de João
Pessoa - PB participaram do estudo. Essa amostra foi não probabilística e sim intencional, 0 propósito era garantir a validade interna dos resultados da pesquisa.

\section{Instrumentos}

Os participantes responderam um questionário composto das seguintes medidas:

Escala de Condutas Antissociais e Delitivas. Este instrumento, proposto por Seisdedos (1988) e validado por Formiga e Gouveia (2003) para 0 contexto brasileiro, compreende uma medida comportamental em relação às Condutas Antissociais e Delitivas. Tal medida é composta por quarenta elementos, distribuídos em dois fatores, como segue: o primeiro envolve as condutas antisociais, em que seus elementos não expressam delitos, mas comportamentos que desafiam a ordem social e infringem normas sociais (por exemplo, jogar lixo no chão mesmo quando há perto um cesto de lixo; tocar a campainha na casa de alguém e sair correndo). 0 segundo fator relaciona-se às condutas delitivas. Estas incorporam comportamentos delitivos que estão fora da lei, caracterizando uma infração ou uma conduta faltosa e prejudicial a alguém ou mesmo à sociedade como um todo (por exemplo, roubar objetos dos carros; conseguir dinheiro ameaçando pessoas mais fracas). Para cada elemento, os participantes deveriam indicar 0 quanto apresentava o comportamento assinalado no seu dia-a-dia. Para isso, utilizavam uma escala de resposta com dez pontos, tendo os seguintes extremos: $0=$ Nunca_e $9=$ Sempre.

A presente escala revelou indicadores psicométricos consistentes identificando os fatores destacados acima; para a Conduta Antisocial encontrou-se um Alpha de Cronbach de .86 e a Conduta Delitiva ou Delinqüente, .92. Considerando a Análise Fatorial Confirmatória, realizada com 0 
Lisrel 8.0, comprovou-se essas dimensões previamente encontradas $\left(\chi^{2} / \mathrm{gl}=1.35\right.$; AGFI $=.89 ; \mathrm{PHI}(\phi)=.79 ; p>.05)$ na análise dos principais componentes (Formiga \& Gouveia, 2003). Neste estudo, essa escala apresentou alfas próximos aos encontrados nos estudo de Formiga (2003; Formiga \& Gouveia, 2003), tanto para Conduta Antisocial (alpha de Cronbach, $a=$ .84) e para Conduta Delitiva ou Delinqüente, $a=.90$ mantendo sua consistência interna. Questionário dos Valores Básicos - QVB. Este instrumento, elaborado e validado por Gouveia (2008), está composto por 18 itens ou valores básicos, agrupados segundo 0 critério de orientação adotado: pessoal, central e social, distribuídos em seis subfunções específicas dos valores: experimentação (emoção, prazer e sexual), realização (êxito, poder e prestígio), existência (estabilidade pessoal, saúde e sobrevivência), suprapessoal (beleza, conhecimento e maturidade), interacional (afetividade, apoio social e convivência) e normativa (obediência, religiosidade $\mathrm{e}$ tradição).

Para responder 0 instrumento 0 participante deverá indicar 0 grau de importância de cada um dos valores tem como um princípio guia na sua vida, utilizando uma escala de resposta de sete pontos, com os seguintes extremos: 1 ("Totalmente não importante") e 7 ("Extremamente importante"). De acordo com as 199 análises fatoriais confirmatórias efetuadas por Gouveia (ver, Gouveia, Milfont, Fischer \& Santos, 2008), este instrumento apresenta índices de bondade de ajuste satisfatórios: $\chi^{2} / g l=2.67 ; \mathrm{GFI}=$ .91; $\mathrm{AGFI}=.89$ e RMSEA $=.05$; sua consistência interna (Alfa de Cronbach) média foi de .51 para o conjunto das seis sub-funções: Existência, Realização, Normativa, Suprapessoal, Experimentação e Interacional.

Caracterização Sócio-Demográfica. Foram elaboradas perguntas que contribuíram para caracterizar os participantes deste estudo (por exemplo, sexo, idade, estado civil, classe social).

\section{Procedimento}

Procurou-se definir um procedimento padrão que consistia em aplicar os instrumentos coletivamente em sala de aula, em escolas em diversas áreas urbanas da cidade de João Pessoa - PB. Desta forma, colaboradores com experiência metodológica e ética, ficaram responsáveis pela coleta dos dados. Após conseguir a autorização tanto da diretoria da escola quanto dos professores responsáveis por cada disciplina, os aplicadores se apresentavam em sala de aula como interessados em conhecer as opiniões e os comportamentos das pessoas no cotidiano, solicitando a colaboração voluntária dos estudantes no sentido de responderem um questionário breve.

Foi-lhes dito que não havia resposta certa ou errada e que, mesmo necessitando de uma resposta individual, estes não deveriam sentir-se obrigados em responder 0 instrumento podendo desistir a qual momento, seja quando tivesse 0 instrumento em suas mãos, ao iniciar sua leitura ou outra eventual condição, bastava apenas contatar as pessoas responsáveis pela aplicação do instrumento no local. A todos era assegurado 0 anonimato das suas respostas, enfatizando que elas seriam tratadas, estatisticamente, em seu conjunto de respostas; apesar do questionário ser auto-aplicável, contando com as instruções necessárias para que possam ser respondidos, os colaboradores estiveram presentes durante toda a aplicação para retirar eventuais dúvidas ou realizar esclarecimentos que se fizessem indispensáveis, não interferindo na lógica e compreensão das respostas dos respondentes; 30 minutos foram suficientes para concluir essa atividade. 
Em relação à análise dos dados da pesquisa, utilizou-se a versão 18.0 do pacote estatístico SPSS para Windows e computadas estatísticas descritivas (tendência central e dispersão). No AMOS 18.0 computaram e avaliaram-se os indicadores estatísticos para o Modelo de Equações Estruturais (SEM) considerando, segundo uma bondade de ajuste subjetiva, os seguintes indicadores: $\chi^{2 / g l}$ (grau de liberdade), que admite como adequados, índices entre 2 e 3, aceitando-se até 5; Raiz Quadrada Média Residual - RMR, indica 0 ajustamento do modelo teórico aos dados, na medida em que a diferença entre os dois se aproxima de zero (0); índices de qualidade de ajuste, dados pelos GFI/AGFI, que medem a variabilidade explicada pelo modelo, e com índices aceitáveis a partir de 0,80; $\mathrm{CFI}$, que compara de forma geral 0 modelo estimado e o modelo nulo, considerando valores mais próximos de um (1) como indicadores de ajustamento satisfatório; $\mathrm{NFI}$, varia de zero a um e pode ser considerado aceitável para valores superiores a 0,90. Caracteriza-se por ser uma medida de comparação entre o modelo proposto e o modelo nulo, representando um ajuste incremental e o RMSEA, que refere-se ao erro médio aproximado da raiz quadrática, deve apresentar intervalo de confiança como ideal situado entre 0,05 e 0,08. (Byrne, 2001; Hair, Tatham, Anderson \& Black, 2005; Joreskög \& Sörbom, 1989).

\section{RESULTADOS}

Sendo o objetivo principal desse estudo, testar um modelo teórico (causal) para explicar as condutas desviantes a partir do construto dos valores humanos, considerouse um modelo recursivo de equações estruturais; a título de lembrança ao leitor, duas hipóteses foram elaboradas: 1) os valores humanos de orientação social associarão, positivamente, a conduta desviante; para comprová-la, gerou-se no AMOS GRAFICS 18.0, o modelo proposto, o qual apresentou o seguinte resultado: $\chi^{2} / \mathrm{gl}(.15 / 1)=.15, p<.70 ; \mathrm{RMR}=.01 ; \mathrm{GFI}$ $=1.00 ; \mathrm{AGFI}=.99 ; \mathrm{NFI}=1.00 ; \mathrm{CFI}=1.00 \mathrm{e}$ RMSEA $=0.00 \quad(.00-.07)$. Os pesos (saturações) que explicam 0 modelo em questão são expostos na figura 1, após as devidas modificações de ajuste, comprovou que a variável independente - 0 construto dos valores humanos de orientação social associou-se, inversamente $(\lambda=-.31)$ as condutas desviantes (composta pela conduta antissocial e delitiva) (Figura 1).

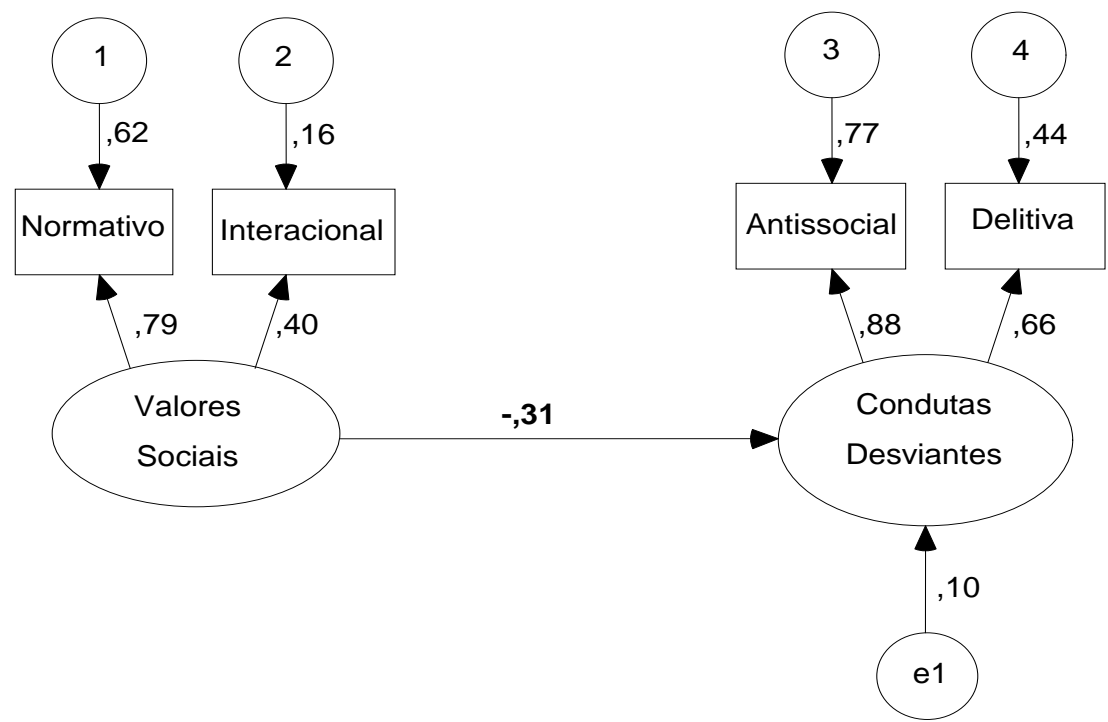

FIGURA 1. Modelagem estrutural da associação entre orientações valorativas sociais e condutas desviantes em jovens. 
A segunda hipótese refere-se à associação positiva dos valores pessoais com as condutas desviantes; desta forma, foi gerado também no AMOS GRAFICS 18.00 modelo proposto, o qual, apresentou 0 seguinte resultado: $\chi^{2} / g l(2.36 / 1)=2,36, p<$ $.12 ; \mathrm{RMR}=.01 ; \mathrm{GFI}=1.00 ; \mathrm{AGFI}=.98 ; \mathrm{NFI}$ $=.99 ; \mathrm{CFI}=1.00$ e RMSEA $=.04(.00-.12)$.

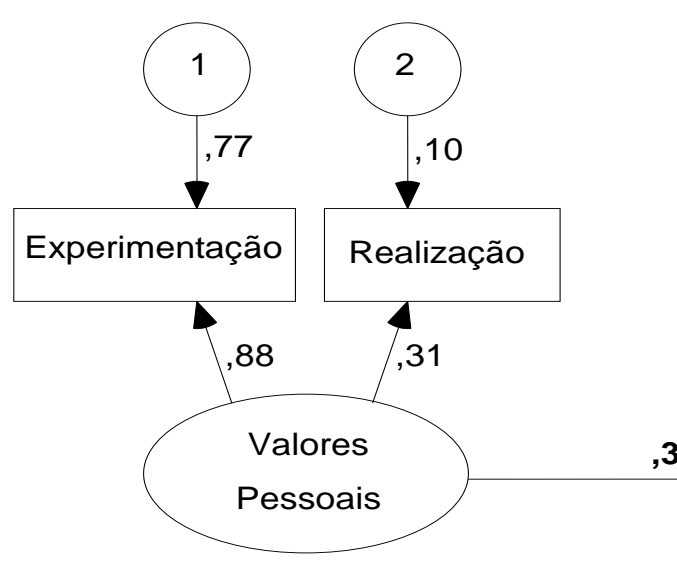

Os pesos (saturações) que explicam 0 modelo em questão são expostos na figura 2, após as devidas modificações de ajuste, comprovou que a variável independente - 0 construto dos valores humanos de orientação pessoal - associou-se, diretamente $(\lambda=.31)$ as condutas desviantes (Figura 2).

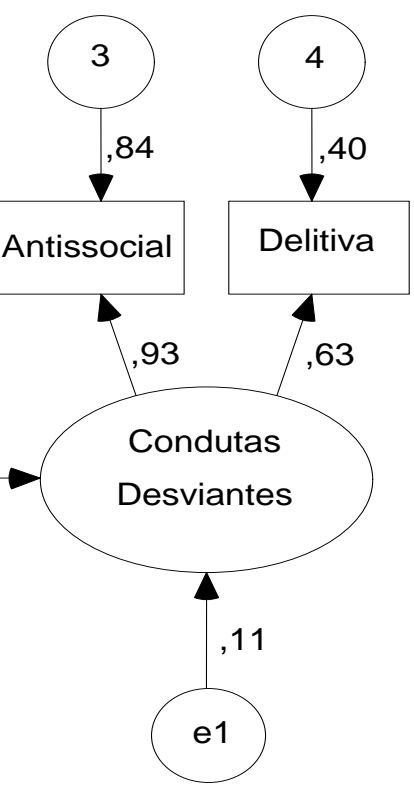

FIGURA 2. Modelagem estrutural da associação entre orientações valorativas pessoais e condutas desviantes em jovens.

Considerando esses resultados, corroboram-se os achados observados por Formiga e Gouveia (2005; 2006). Estes estudos foram à base para modelagem empírica do presente estudo, bem como, fornece indicadores estatísticos mais robustos em termos das estruturas associativas entre as variáveis, provando que dependendo da orientação valorativa que os jovens venham a aderir, provavelmente, manifestarão ou não a conduta desviante. Por exemplo, aquele jovem que pontue mais alto em valores sociais, menor será a sua conduta desviante, enquanto uma pontuação maior em valores pessoais, maior a conduta desviante.

Tomando a base teórica de que os valores, além de ser desejável por cada sujeito, afinal, não se toma decisão ou se faz escolhas no vazio social, guiam a seleção ou avaliação de comportamentos e eventos e representam cognitivamente as necessidades humanas. Neste contexto, 0 presente trabalho, procurou oferecer um referencial que permita uma intervenção nos valores humanos de modo a inibir as condutas anti-sociais e delitivas entre os jovens.

Conhecer um padrão valorativo de um fenômeno social desviante, especificamente, 0 da conduta juvenil antissocial e delituosa é apontar em direção de um modelo teórico consistente a fim de compreender não somente sua capacidade preditiva dessa variável, mas também, de uma melhor direção conceitual aplicada em relação às políticas publicas para a juventude. 


\section{CONCLUSAO}

Nos estudos anteriormente citados sobre as condutas desviantes (Coelho Junior, 2001; Formiga, 2002; Formiga \& Gouveia, 2005) é evidente que estas condutas referem-se à debilidade dos limites convencionais que vão desde a falta e adesão de um comprometimento com as convenções exigidas pela sociedade e suas instituições de controle social frente aos valores assumidos pelas pessoas que a compõem.

Por exemplo, Formiga (2010; Formiga, 2005) observaram que uma maior afiliação dos jovens aos pais (pai e/ou mãe) e professores, menor a conduta antissocial e delitiva. Esse fato pode ser compreendido em relação ao envolvimento dos jovens com a sociedade convencional tornando-os capaz de internalizar os valores ou padrões convencionais, comportando-se segundo as normas sociais vigentes (Formiga, 2002).

Apesar da consistência desses resultados, alguns limites podem ser destacados para futuras pesquisas: 1- seria de importante a construção e testagem de um modelo teórico capaz de provar a associação entre valores humanos, afiliação com os pares sócio-normativos e conduta desviante; 2 - outro estudo, também, significativo seria a associação entre valores humanos, práticas parentais e conduta desviante. Tais variáveis poderiam corroborar a hipótese da internalização dos valores a partir da dinâmica socializadora da família.

\section{REFERENCIAS}

Agüero, A. J. (1998). El transtorno de conducta en la infancia como precursor del trastorno antisocial del adulto. Estudios de seguimiento a medio y largo plazo. Necesidad de programas preventivos. Revista Electrónica de Psiquiatria ${ }_{2} 2,1-9$.

Byrne, B. M. (1989). A primer of LISREL: Basic applications and programming for confirmatory factor analytic models. New York: Springer-Verlag.
Coelho Junior, L. L (2001). Uso pontencial de drogas em estudantes do ensino médio: Suas correlações com as prioridades axiológicas. Dissertação de Mestrado. Departamento de Psicologia Social. Universidade Federal da Paraíba.

Farias, S. A. y Santos, R. C. (2000). Modelagem de Equações Estruturais e Satisfação do Consumidor: Uma Investigação Teórica e Prática. Revista de Administração Contemporânea, 4 (3), 107-132.

Formiga, N. S. (2002). Condutas anti-sociais e delitivas: Uma explicação baseada os valores humanos. Dissertação (Mestrado em Psicologia Social). Universidade Federal da Paraíba.

Formiga, N. S. (2003). Fidedignidade da escala de condutas anti-sociais e delitivas ao contexto brasileiro. Psicologia estudo, 8 (2), 133-138.

Formiga, N. S. (2005). Comprovando a hipótese do compromisso convencional: Influência dos pares sócio-normativos sobre as condutas desviantes em jovens. Revista psicologia ciência e profissão, 25 (4), 602613.

Formiga, N. S. (2006). Valores humanos e condutas delinqüentes: As bases normativas da conduta anti-social e delitiva em jovens brasileiros. Psicología para América Latina. , 7, 1 -15.

Formiga, N. S. (2010). Pares sócio-normativos e condutas desviantes: Testagem de um modelo teórico. Barbarói, 32 (1), 43-20.

Formiga, N. S. y Diniz, A. (2011). Estilo da orientação cultural e condutas desviantes: Testagem de um modelo teórico. Revista Pesquisa em Psicologia, 5 (1). Manuscrito o prelo.

Formiga, N. S. y Gouveia, V. V. (2003). Adaptação e validação da escala de condutas anti-sociais e delitivas ao contexto brasileiro. Revista Psico, 34 (2), 367-388.

Formiga, N. S. y Gouveia, V. V. (2005). Valores humanos e condutas anti-sociais e delitivas. Psicologia: teoria e prática, 7 (2), 134-170.

Gouveia, V. V.; Milfont, T. L.; Fischer, R. y Santos, W. S. (2008). Teoria funcionalista dos valores humanos. Em: TEIXEIRA, Maria L. M. Valores humanos e Gestão: novas perspectivas. (p. 47-80). São Paulo: Ed. Senac. 
Hair, J. F.; Tatham, R. L.; Anderson, R. E. y Black, W. (2005). Análise Multivariada de Dados. Porto Alegre: Bookman.

Joreskög, K. \& Sörbom, D. (1989). LISREL 7 user's reference guide. Mooresville: Scientific Software.

Lipovetsky, G. (1986). La era del vacío: Ensayos sobre el individualismo contemporáneo. Barcelona: Editorial Anagrama.

Muñoz-Rivas, M. y Graña, J. L. L. (2002). Factores familiares de riesgo y de protección para el consumo de drogas en adolescentes. Psicothema, 13, n. 1, 87-94.

Petraitis, J.; Flay, B. R. \& Miller, T. Q. (1995). Reviewing theories of adolescent substance use: Organizing pieces in the puzzle. Psychological Bulletin _117, 67-86.

Romero, E.; Sobral, J., Luengo, M. A. \& Marzoa, J. A. (2001).Values and antisocial behavior among Spanish adolescents. The Journal of Genetic Psychology, 162, 20-40.

Seisdedos, N. C. (1998). Cuestionario A-D de conductas antisociais - delictivas. Madri: TEA. 1988.

Silva, J. S. F. (2006). Modelagem de Equações Estruturais: Apresentação de uma metodologia._Dissertação (Mestrado em Engenharia de Produção). Universidade Federal do Rio Grande do Sul. Recuperado em 25 de agosto de 2009, da WEB (página da WEB): http://hdl.handle.net/10183/8628.

Stoff, D. M.; Breiling, J. \&_Maser, J. D. (1997). Handbook of Antisocial Behavior. Canada: John Wiley and Sons.

Sukhodolsky, D. G.; Colub, A. G. \&_Cromwell, E. N. (2001). Development and Validation of the Anger Rumination Scale. Personality and Individual Differences, 31 (5), 689-700.

Tamayo, A.; Nicaretta, M., Ribeiro, R. y_Barbosa, L. P. G. (1995). Prioridades axiológicas y consumo de drogas. Acta Psiquiátrica y Psicológica de la América Latina, 4, 300307.

Torrente H. G. y Rodríguez G. Á. (2000). Precedentes sociofamiliares de la conducta antisocial. In: A. O. Bernal, M. V. M. Jiménez; P. V. i Elias (Eds.), Aplicaciones en psicología social. (p. 197202). Madri: Biblioteca Nueva. 